FORMATION Formation emploi

Revue française de sciences sociales

134 | Avril-Juin

Pêle-mêle

\title{
Les figures de réussite, des clés pour appréhender les bifurcations scolaires et professionnelles
}

Social models of success. Keys to comprehending bifurcations in school and work trajectories

Erfolgsbilder, Schlüssel zum vorgreifenden Verständnis der schulischen und beruflichen Richtungswechsel

Los casos de éxito, claves para aprehender las bifurcaciones escolares y

profesionales

Sylvain Bourdon, María-Eugenia Longo et Johanne Charbonneau

\section{(2) OpenEdition}

Journals

Édition électronique

URL : http://journals.openedition.org/formationemploi/4718

DOI : 10.4000/formationemploi.4718

ISSN : 2107-0946

Éditeur

La Documentation française

Édition imprimée

Date de publication : 21 juin 2016

Pagination : 27-45

ISSN : 0759-6340

Référence électronique

Sylvain Bourdon, María-Eugenia Longo et Johanne Charbonneau, «Les figures de réussite, des clés pour appréhender les bifurcations scolaires et professionnelles », Formation emploi [En ligne], 134 | Avril-Juin, mis en ligne le 23 juin 2018, consulté le 30 octobre 2020. URL : http://

journals.openedition.org/formationemploi/4718; DOI : https://doi.org/10.4000/formationemploi.4718

(c) Tous droits réservés 


\title{
Les figures de réussite, des clés pour appréhender les bifurcations scolaires et professionnelles
}

\author{
SYLVAIN BouRdon \\ Sociologue, Faculté d'éducation de l'université de Sherbrooke (Québec) et Centre d'études et \\ de recherches sur les transitions et l'apprentissage (CÉRTA) \\ MARÍA-Eugenia Longo \\ Sociologue, Centre Urbanisation Culture Société - INRS (Québec) et Laboratoire d'économie \\ et de sociologie du travail (LEST - CNRS), université Aix-Marseille \\ Johanne Charbonneau \\ Sociologue, Centre Urbanisation Culture Société - INRS (Québec)
}

Résumé

Les figures de réussite, des clés pour appréhender les bifurcations scolaires et professionnelles

Malgré leur complexification croissante, les parcours d'insertion des jeunes continuent d'être le résultat d'un processus de socialisation qui, plutôt que de s'ancrer dans un modèle unique, reflète une cohabitation de normes plus ou moins contradictoires. Les figures de réussite ont été élaborées et mises à l'épreuve dans l'analyse d'entretiens longitudinaux menés auprès de 53 jeunes Québécois. Il s’agissait de mieux comprendre le rôle des référents normatifs dans les choix opérés, leur enchaînement dans les parcours d'insertion, et les bifurcations qui peuvent en résulter.

Mots clés : jeune, trajectoire d'insertion, orientation scolaire-professionnelle, Québec

Abstract

Social models of success. Keys to comprehending bifurcations in school and work trajectories

The increasing complexification of youth's pathways to adulthood reflects a shift from a socialization process rooted in relatively homogenous social influences to a cohabitation of multiple, and often contradictory, social norms. Social models of success have been extracted and tested in the analysis of longitudinal interviews with 53 young Quebecers to better comprehend the role played by normative references in the choices they made, in their sequencing along the lifecourse and in the bifurcations they fostered. 
Keywords: young person, pathway to integration, school guidance \& vocational guidance, Quebec

Journal of Economic Literature: J 29, J 24

Traduction : Auteur.e.s.

Depuis quelques décennies, on assiste à l'individualisation des inégalités sociales dans nos sociétés du risque (Beck, 2001) et de l'incertitude, où les responsabilités et les aléas, naguère assumés par les collectifs - État, entreprises, organisations, famille - sont de plus en plus pris en charge par les individus. Dans ce contexte, la différenciation et la complexification croissante des parcours d'insertion des jeunes ont attiré l'attention des observateurs dans plusieurs pays : choisir un programme scolaire, enchaîner des boulots étudiants, s'engager rapidement ou non dans une carrière. Malgré leur complexification et leur différenciation croissante, les parcours d'insertion des jeunes ne sont pas pour autant aléatoires et dénués de tout ancrage social. Comme le souligne Cavalli (2007, p.67) «Le modèle de parcours de vie en vigueur garde son caractère normatif; il est cependant plus diffcile à accomplir sur le plan individuel ». Ainsi, les parcours continuent d'être le résultat d'un processus de socialisation qui, plutôt que de s'ancrer dans un modèle unique, reflète de plus en plus une cohabitation de normes plus ou moins contradictoires. Devant une telle situation, le sociologue peut tenter de formuler, à partir de sa perspective, une norme de réussite à l'aune de laquelle il pourra juger de l'efficacité des diverses filières ou de celle des stratégies individuelles observées. Mais il peut aussi s'intéresser à la définition même de cette norme de réussite du point de vue du sujet, afin de mieux comprendre son effet sur les choix effectués et leur enchaînement dans les parcours d'insertion.

Un travail collectif nous a conduit à nous interroger sur les références qui fondent, tant chez le sociologue que chez le jeune interviewé, le constat d'une bifurcation. Ce travail portant sur les bifurcations visait à mettre au jour, sur la base de données qualitatives longitudinales, le sens des actions qui composent les parcours (Bessin, Bidart et Grossetti, 2010), pour en dégager, au-delà des singularités, les logiques sociales qui surplombent et orientent les décisions.

Compte tenu des divergences, tant dans nos bases de classification que dans les discours des jeunes adultes dans des situations comparables, il est apparu nécessaire de réinterroger les fondements normatifs mobilisés dans l'élaboration même du construit de bifurcation. Quand les jeunes bifurquent, par rapport à quoi le font-ils ? Un changement radical de

1. L'analyse à l'origine de cette réflexion s'est amorcée dans le cadre d'un projet qui rassemblait des équipes argentine, française et québécoise dans un effort de comparaison internationale : Claire Bidart (dir.) «BIPAJE. La bifurcation biographique au cour de la dynamique des parcours d'entrée de la vie professionnelle " (ANR-09-BLAN-0301-01). Une version préliminaire de cet article a été présentée aux XXI ${ }^{\grave{e}}$ journées du longitudinal, Dijon, IREDU-université de Bourgogne, en décembre 2015. 
parcours constitue-t-il toujours une bifurcation, indépendamment des modèles des jeunes ou du contexte sociétal dans lequel il s'effectue ? Le construit de figure de réussite s'est ainsi avéré fécond pour mieux comprendre les choix qui définissent les parcours d'insertion et leurs changements.

Ces figures de réussite forment des idéaux-types - au sens webérien du terme - des conceptions véhiculées dans l'espace social et auxquelles se rapportent les individus pour imaginer et sélectionner les possibilités qui s'offrent à eux en termes de formation, de travail et de place du travail dans leur vie. On peut penser, par exemple, à la figure de l'entrepreneur, sur laquelle nous reviendrons plus loin, pour laquelle les possibilités de mobilité sociale et l'encouragement de l'initiative individuelle priment sur l'investissement dans des études longues et sur la sécurité de l'emploi. Parce qu'elles constituent des " incarnations » relativement tangibles de cibles à atteindre, témoignant de leur désirabilité dans un contexte social donné, ces figures de réussite permettent de "remonter ", à partir des parcours individuels, vers les normes propres à un contexte donné.

Après un bref exposé méthodologique, nous présentons les différentes figures de réussite reconstruites à partir des discours des jeunes, en les associant à certaines positions et dimensions particulières du contexte sociétal québécois, dans lequel elles s’inscrivent. Un examen de la concomitance et des changements de figures relevés dans certains parcours permettra ensuite d'analyser les liens entre les changements de représentations de la réussite chez les jeunes et les changements ou bifurcations observés « de l'extérieur » dans leurs parcours.

\section{Une reconstruction des figures de réussite à partir du discours des jeunes}

Les parcours de vie en général, et les parcours professionnels en particulier, sont constitués par des séquences de comportements (accepter ou non un emploi ; poursuivre ou non ses études dans un domaine particulier,...). Alors qu'on peut leur trouver suffisamment de ressemblances pour les grouper en types ou les étudier de manière agrégée, les parcours demeurent individuels et singuliers.

L'analyse sociologique des bifurcations biographiques tire sa pertinence de l'idée selon laquelle les suites de décisions individuelles qui construisent les parcours ne sont ni totalement déterminées ni totalement libres. Même si elle conçoit les parcours comme résultant d'une séquence de choix, cette analyse ne postule toutefois pas une rationalité parfaite des acteurs. Elle adhère plutôt à une hypothèse de rationalité limitée, l'information dont disposent les individus étant nécessairement incomplète et interprétée à partir de leurs dispositions particulières, elles-mêmes fonction de leur position dans l'espace social. Pour le dire plus abruptement, tous les choix ne sont pas aussi accessibles à tous, notamment parce que l'accès à l'information ou aux conceptions est inégalement réparti entre les 
groupes sociaux. Dans ce cadre, l'orientation normative de l'action renvoie ainsi à la poursuite d'un "terme perçu comme favorable ", sans toutefois limiter ce caractère favorable à une simple finalité égoïste (Demeulenaere, 2003) d'optimisation de la satisfaction individuelle. Dès lors, on s'écarte des postulats théoriques de l'économie classique qui reposent sur l'unique maximisation du profit ou de la satisfaction, pour envisager des individus pouvant se référer à des visées variées (variations interindividuelles) et diversifiées (variations intra-individuelles); ces visées pouvant se modifier dans le temps pour un même individu. Sans réifier la notion de projet ou nier l'existence de positions sociales particulièrement aliénantes (au sens où les individus y sont étrangers à eux-mêmes, sans prise agentique réelle), il nous apparaît aussi important, pour opérationnaliser l'analyse des parcours, de postuler que ces choix visent (sans préjuger du réalisme ou de l'atteinte de ce but) une forme d'accomplissement (Sen, 2000) perçue comme désirable, et particulièrement de ce que constitue un travail satisfaisant et de la place qu'il devrait occuper dans l'ensemble de la vie.

Ainsi, les choix et décisions - ainsi que les non-choix et les indécisions - qui jalonnent les parcours d'insertion des jeunes sont opérés en fonction de la lecture qu'ils font de leur situation à un moment donné et de certaines aspirations plus ou moins clairement définies (Longo, Bourdon, Charbonneau, Kornig et Mora, 2013). Ces projections individuelles ne se forment pas ex nibilo, mais dans le cadre d'une société qui offre différents modèles de réussite professionnelle et sociale, historiquement construits sur des agencements spécifiques de normes plus ou moins valorisées, répandues et institutionnalisées. C'est par leur caractère social que les normes et les modèles sont susceptibles de se propager et que nous pouvons en observer les traces au sein des récits des jeunes.

C'est ce que nous avons fait dans le cadre de l'analyse d'une enquête réalisée au Québec par Sylvain Bourdon et Johanne Charbonneau, auprès de jeunes de 17 à 19 ans ayant amorcé leurs études dans deux cégeps, ${ }^{2}$ en $2003^{3}$. Les 53 jeunes adultes retenus pour la présente analyse proviennent de deux établissements, un à Montréal et l'autre à Sherbrooke. Six vagues d'entretiens ont eu lieu : automne $2004(\mathrm{n}=53)$; printemps $2005(\mathrm{n}=53)$; automne $2005(\mathrm{n}=51)$; printemps $2007(\mathrm{n}=35)$; printemps $2008(\mathrm{n}=29)$ et printemps $2009(\mathrm{n}=20)$, soit un total de 241 entretiens. Chaque vague était l'occasion de dresser un inventaire du réseau social, un calendrier des cycles de vie situant mois par mois leur parcours dans les principales sphères de vie, et de participer à un entretien qualitatif abor-

2. Les cégeps (collèges d'enseignement général et professionnel) sont des établissements d'enseignement postsecondaire qui ont vu le jour au Québec, à la fin des années 1960. L'idée fondatrice était de faire évoluer, dans un même établissement, des jeunes en formation technique (parcours de trois ans destinant au marché du travail) et en formation préuniversitaire (deux ans préparatoires à l'université, non terminal en principe) pour favoriser le brassage social et le "réchauffement " (Bélanger, 1986) des ambitions scolaires d'une majorité canadienne-française à l'époque relativement sous-scolarisée.

3. Projet financé par le Fonds québécois de recherche sur la société et la culture (FQRSC) et le ministère de l'Éducation, du Loisir et du Sport du Québec (MELS). 
dant divers aspects de leur parcours et choix scolaires et professionnels. Ces entretiens ont été transcrits et codés à l'aide du logiciel NVivo, selon une grille d'analyse permettant de cibler, dans la masse des données recueillies, celles portant sur l'un ou l'autre des thèmes abordés lors des entretiens.

L'analyse des figures de réussite a mobilisé l'ensemble des entretiens qualitatifs, en se concentrant particulièrement ${ }^{4}$ sur les extraits codés aux thèmes agrégeant les représentations des études, du travail et des projets futurs. Le processus s'est effectué en trois grandes itérations qui ont permis d'articuler de plus en plus précisément l'empirique et la théorie. La première itération a permis de tracer les grandes lignes des figures de réussite à partir d'une lecture globale de l'ensemble du corpus. Il ne s'agit pas ici d'une analyse lexicométrique ou automatisée, mais d'une analyse sociologique assez classique visant l'élaboration d'une typologie qualitative à partir d'une comparaison des discours des acteurs. Cette analyse a fait émerger quatre figures qui correspondent d'assez près aux figures 1, 2, 3 et 5 proposées dans la partie suivante. Les principales propriétés de ces figures ont alors fait l'objet d'un exercice d'explicitation et de corrélation avec certaines caractéristiques du contexte sociétal québécois, par exemple la valorisation des formations axées sur la professionnalisation et la figure du professionnel diplômé.

La deuxième itération analytique a tenté d'associer une figure à chaque vague pour chacun des jeunes, dans le cadre d'une relecture complète du matériel ciblé. Cette opération de systématisation a conduit à raffiner encore davantage les distinctions entre les figures et à valider leur exhaustivité dans le corpus étudié. Des nuances ont ainsi été apportées qui ont fait se distinguer la figure 6 (éternel étudiant) de la figure 1 (professionnel diplômé) ainsi que la figure 4 (travailleur en sécurité) de la figure 3 (consommateur hédoniste). Les définitions des figures et les critères ont été revus et précisés pour tenir compte de ces ajouts. À la fin de cette étape, nous disposions d'une définition de chacune des figures permettant d'en saisir les traces dans le discours des jeunes et de critères permettant de la distinguer des cinq autres. C'est à ce moment que sont apparus les premiers indices de concomitances et de changements de figures de réussite dans certains parcours.

Pour la troisième et dernière itération, une autre analyste a repris l'ensemble du matériel pour revoir et corriger la classification des observations à l'aide des définitions finalisées, afin d'assigner une ou des figures de référence à chaque entrevue. Le résultat de cette dernière phase, présenté dans le Tableau $\mathbf{1}$, a permis de comptabiliser la prévalence des figures dans l'échantillon et d'y recenser les concomitances et changements de figure.

4. Les « nœuds " du logiciel NVivo servent à regrouper des extraits du corpus portant sur un même thème pour les examiner de manière approfondie dans le cadre d'une analyse particulière, tout en permettant de resituer ces extraits dans leur contexte, au besoin. Notre analyse s'est ainsi concentrée " particulièrement » sur certains extraits, mais sans omettre de les resituer dans le contexte plus large de l'entretien, lorsque cela s'est avéré nécessaire pour une meilleure compréhension de la situation. 


\section{Les six figures de réussite}

Le travail d'analyse a permis d'identifier six figures de réussite dans les discours des jeunes rencontrés. Le Tableau 1 les synthétise brièvement, puis chacune est exposée avant que soient examinés leur distribution dans l'échantillon étudié et les changements dans le temps qui ont pu être observés.

Tableau 1 - Synthèse des principales caractéristiques des figures de référence

\begin{tabular}{|c|c|c|c|}
\hline Figure & $\begin{array}{l}\text { Normes scolaires et } \\
\text { professionnelles }\end{array}$ & $\begin{array}{l}\text { Origine sociale } \\
\text { typique }\end{array}$ & Inscription sociétale \\
\hline $\begin{array}{l}\text { 1. Professionnel } \\
\text { diplômé }\end{array}$ & $\begin{array}{l}\text { Adéquation emploi-diplôme ; primauté } \\
\text { au diplôme }\end{array}$ & $\begin{array}{l}\text { Varie selon le niveau de } \\
\text { diplôme }\end{array}$ & $\begin{array}{c}\text { Valorisation des formations axées sur la } \\
\text { professionnalisation ; soutien financier aux } \\
\text { études (bourses, prêts) }\end{array}$ \\
\hline $\begin{array}{l}\text { 2. Travailleur } \\
\text { identitaire }\end{array}$ & $\begin{array}{l}\text { Se former pour se préparer au travail ; le } \\
\text { travail pour se réaliser ; importance de la } \\
\text { tâche, du métier }\end{array}$ & Plutôt classe moyenne & $\begin{array}{l}\text { Injonction à « être soi » ; marché du travail } \\
\text { ouvert et accessible }\end{array}$ \\
\hline $\begin{array}{l}\text { 3. Consommateur } \\
\text { hédoniste }\end{array}$ & $\begin{array}{l}\text { Le revenu avant tout ; études et emplois } \\
\text { comme des moyens }\end{array}$ & Classe moyenne & $\begin{array}{l}\text { Injonction à la consommation; socialisation à } \\
\text { la consommation par l'emploi étudiant }\end{array}$ \\
\hline $\begin{array}{l}\text { 4. Travailleur en } \\
\text { sécurité (d'emploi) }\end{array}$ & $\begin{array}{c}\text { Sécurité de l'emploi ; études instrumen- } \\
\text { tales pour emploi stable aux conditions } \\
\text { avantageuses }\end{array}$ & $\begin{array}{l}\text { Classes moyenne et } \\
\text { populaire }\end{array}$ & $\begin{array}{c}\text { Certaines possibilités de sécurisation des } \\
\text { parcours (fonction publique, grande entre- } \\
\text { prise, carrière militaire) }\end{array}$ \\
\hline 5. Entrepreneur & $\begin{array}{l}\text { Responsabilité et initiative individuelle } \\
\text { privilégie l'expérience et le « concret » }\end{array}$ & $\begin{array}{l}\text { Classes populaires et } \\
\text { première génération au } \\
\text { postsecondaire }\end{array}$ & $\begin{array}{l}\text { Possibilités de mobilité sociale ; encourage- } \\
\text { ment de l'initiative individuelle }\end{array}$ \\
\hline 6. Éternel étudiant & $\begin{array}{c}\text { Priorité à la vie intellectuelle et à } \\
\text { l'autonomie ; faible priorité à l'insertion } \\
\text { en emploi }\end{array}$ & $\begin{array}{l}\text { Classes moyenne-sup. et } \\
\text { moyenne }\end{array}$ & $\begin{array}{l}\text { Idéalisation des études longues; soutien } \\
\text { financier aux études (famille) }\end{array}$ \\
\hline
\end{tabular}

Sources : Auteur.e.s.

\subsection{Le professionnel diplômé}

Cette figure manifeste un rapport d'adéquation relativement étroit entre un diplôme et un emploi. L'obtention du diplôme est le sésame qui ouvre la porte à des emplois bien identifiés et valorisés socialement. Plus le diplôme sera élevé, plus les conditions d'emploi seront bonnes. La figure du professionnel diplômé sous-tend l'idée d'avancement progressif en carrière.

C'est une figure assez répandue et qui traverse diverses classes sociales. Cependant, les jeunes des différentes classes ne viseront pas les mêmes professions, les plus prestigieuses étant recherchées par les classes supérieures. Cette figure est liée à la confiance dans un système scolaire permettant à chacun de développer ses talents et ses compétences pour accéder à la carrière de son choix. Au Québec, l'accès aux études est facilité par divers types de soutien, notamment un système de prêts et bourses d'études qui financent à la fois les frais directs et indirects de la participation scolaire. 
On retrouve de nombreux exemples de cette figure de réussite, notamment celui de Kevin, qui poursuit des études collégiales dans le but clairement affiché de devenir comptable agréé. Cet objectif est associé à la fois à l'évolution sociale de sa famille et au goût pour ce domaine. Les études ont alors la priorité et les séquences planifiées pour sa carrière sont assez définies : d'abord les études, ensuite l'emploi en correspondance avec le diplôme, enfin l'évolution dans le même domaine. C'est pour cette raison qu'il ne travaille pas lors des premières vagues, choix soutenu par ses parents qui l'encouragent à se concentrer sur les études. Ce n'est qu'un peu plus tard qu'il occupera des emplois étudiants temporaires en lien avec son domaine de formation, sous forme d'un stage en alternance études-travail (vague 2), puis comme caissier dans une banque (vague 3), ou en tant que commis dans un bureau (vague 4). Ces emplois étudiants ne remettent toutefois jamais en cause son objectif principal d'obtenir les diplômes, collégial puis universitaire, qui lui permettront de gravir des échelons dans sa profession :

"Q: Tes études en techniques administratives, tu les fais dans quel but?"

"Bien, pour devenir comptable agréé. Parce que je veux faire l'université après là. [...] J'aime les chiffres là."

"Q: Tu te vois où dans cinq ans?"

"Sûrement dans une firme de comptables ou peut-être partir à mon compte " (vague 1)

\subsection{Le travailleur identitaire}

Pour le travailleur identitaire, le contenu de la tâche prime ; les activités définissant l'emploi doivent correspondre le plus étroitement possible à l'identité que le jeune construit en avançant en âge.

Cette figure repose sur l'idée qu'il est toujours possible de trouver un emploi correspondant à ses goûts, ses aspirations, l'important étant de bien se connaître, de réfléchir et de choisir. Elle repose sur la représentation d'un marché du travail relativement ouvert qui permet à chacun de réaliser ses désirs. Le travail doit rendre heureux ; l'emploi est la première source d'épanouissement personnel. Cette figure s'est construite en même temps que se diversifiait l'offre de programmes d'études supérieures, au gré des initiatives des institutions d'enseignement. Elle dépend de deux conditions : 1) un coût des études relativement bas pour tous les programmes, y garantissant un accès ouvert ; 2) des parents encourageant l'individualité de leurs enfants et leur liberté de choix. C'est une figure répandue dans l'ensemble des classes, avec une certaine prépondérance au sein de la classe moyenne.

L'exemple de Rebecca illustre cette figure. D'abord engagée dans des études d'art-thérapie, elle envisage plus tard de devenir enseignante, sans vraiment définir un domaine d'enseignement particulier. Pour elle, de toute façon, ce qui compte, ce sont l'épanouissement et 
la passion avec laquelle on envisage la tâche, une valorisation qui s'affirme à mesure que le parcours progresse. Le modèle paternel renforce ici l'inscription dans cette figure :

"Je vois que mon père est épanoui à fond là-dedans, et c'est ça que je trouve le fun, et c'est ça que j'ai le goût de reproduire. Tout ce que ça m'a dit moi, dans le fond, c'est que je ne veux pas une vie de bourreau de travail, du genre qui fait quelque chose qu'il n'aime pas. [...] Parce que je me dis que ce serait ridicule de gâcher ma vie... Tu sais, on passe quand même la plus grande partie de notre vie à travailler, fait que pourquoi faire de quoi qui t'intéresse pas, pis, pourquoi continuer à le faire quand ça t'intéresse pas? C'est pour ça que je veux vraiment faire de quoi que j'aime et que je vais changer si ça m'intéresse pas. Comme lui dans le fond. [...] Je veux tripper moi, c'est ça pour moi, je veux tripper. Puis je veux être passionnée, puis j’veux aimer ça [...] Le plus important dans le travail ? Ben la passion là. " (vague 1)

\subsection{Le consommateur hédoniste}

Cette figure s'inscrit en réponse directe aux injonctions d'une société de consommation; l'accent étant mis sur le salaire associé à l'emploi qui doit permettre de satisfaire en priorité les désirs d'une consommation hédoniste.

Chez les jeunes Québécois, cette figure, influencée par les sollicitations commerciales qui encouragent la surconsommation, prend aussi sa source dans le développement massif de l'emploi étudiant qui a donné accès à un budget discrétionnaire servant d'abord à se payer du superflu. On peut penser que c'est la volonté de maintenir ce niveau de dépenses hédonistes, alors que s'y ajouteront un jour les dépenses de base souvent assumées par les parents pendant les études, qui a construit cette figure de réussite. Si la "surconsommation ", bien que de nature différente, est répandue dans toutes les classes, on peut rattacher cette figure à la classe moyenne, où les revenus de la famille s'additionnent souvent à ceux obtenus par les jeunes eux-mêmes grâce aux emplois étudiants.

Le cas de Julien illustre cette figure tout au long des vagues. Son but est clair : travailler pour "ramasser de l'argent" autour duquel tout le reste (logement, formation, couple, famille) s'organisera. Après un passage par l'armée en vague 1 , où il envisage une carrière qui lui permettrait d'esquiver les études, il abandonne toute ambition scolaire pour se concentrer sur l'emploi, essentiellement en vue de jouir d'un revenu plus conséquent et immédiat, même si cela le cantonnera à enchaîner des emplois instables et moins qualifiés :

"Q: Qu'est-ce que ça veut dire pour toi travailler?"

"Bien, faire de l'argent pour acheter des affaires. " (vague 1)

"J'ai décidé de lâcher l'armée à cause des dettes que j'avais. À 17 ans, avec un taux élevé de dettes, c'est pas normal. Je trouvais qu'avec l'armée, ça faisait pas assez d'argent. [...]" 
"Q: Tes rêves, tes aspirations aujourd'hui??"

"Devenir architecte, puis, c'est comme je t'ai expliqué tantôt, une grosse maison, une grosse auto, une piscine creusée en arrière... Une belle vie quoi. " (vague 2)

\subsection{Le travailleur en sécurité (d'emploi)}

On pourrait l'appeler la figure du fonctionnaire, mais l'emploi dans l'administration publique n'est pas seul à y donner accès, car plusieurs grandes entreprises syndiquées offrent des perspectives d'emploi presque aussi stables et avantageuses du point de vue des conditions d'emploi. Le contenu du travail importe peu ; c'est le sentiment de sécurité à long terme qui prime. Cette figure est valorisée par les classes moyenne et populaire, et plus particulièrement par ceux qui ont conscience d'une certaine limite dans leur capacité à poursuivre de longues études ou qui sont déçus par une formation spécifique, tout en ayant bon espoir de s'inscrire dans une certaine mobilité sociale.

C'est le cas de Simon qui, dès notre première rencontre, cherche un moyen d'assurer son parcours " jusquà la retraite ». La sécurité de l'emploi est posée comme le principal moteur de son insertion et il fait tout pour l'atteindre, en se destinant à une carrière militaire qui offre de bonnes conditions de stabilité, de sécurité et de progression dans le secteur de l'électronique. Les études constituent pour lui un moyen d'atteindre son but :

"Au début, tu signes un contrat de trois ans. Tu peux signer un contrat de 20 ans, comme certains ont fait, ou tu peux signer encore des contrats de trois ans tout le temps. C'est parce que l'affaire, dans l'armée, c'est que maintenant ça prend 25 ans et tu peux prendre ta retraite." (vague 2)

\subsection{L'entrepreneur}

La figure de l'entrepreneur s'appuie sur l'idée que le succès dépend de l'initiative individuelle, de la débrouillardise, de l'ingéniosité, plus que de l'héritage familial. Elle valorise la possibilité de créer soi-même son emploi et d'être son propre patron. Cette figure est aussi associée à l'idée de l'effort et de la prise de risque. En ce sens, la trajectoire qui permet d'atteindre la cible peut être complexe (un « bricolage résilient ») et semée d'embûches. La version nord-américaine de cette figure - celle que l'on retrouve au Québec - s'appuie sur la notion de mobilité sociale, selon laquelle on peut partir du bas de l'échelle sociale et progresser vers le sommet. Sans tout à fait nier le poids du diplôme, cette figure en relativise l'importance en l'intégrant à l'éventail des autres ressources valorisées (l'expérience, les contacts,...). C'est une figure associée à la classe populaire et aux jeunes qui constituent la première génération dans la famille à obtenir un diplôme et qui valorisent davantage l'expérience pratique et concrète que les connaissances transmises dans le cadre scolaire.

L'exemple de Zachary illustre l'esprit entrepreneurial mis à disposition de la construction du parcours. L'autonomie et la progression sociale associées à l'emploi apparaissent de 
façon claire, ainsi que le caractère accessoire du diplôme ou l'importance des contacts pour faire avancer les projets. L'importance accordée au fait de créer lui-même son emploi (propre organisme, propre entreprise), la grande flexibilité quant au secteur d'activité visé (soutien à la communauté, cuisine, sport) et l'ouverture à la saisie d'opportunités apparaissent tout au long des vagues et illustrent la sensation de maitrise du parcours face aux changements :

"Je me suis dit que jaimerais ça un jour, monter mon propre organisme, pas une entreprise là... Mener mes projets à terme aussi. Je me suis dit que le [cégep] me donnerait une certaine crédibilité en fait, une certaine confiance, puis mouvrirait des portes dans mon cheminement. [...] Ça fait que, tu sais, je vais attendre de voir le cours qui soit me branche le plus ou soit que j’ai une facilité à développer mes forces et mes faiblesses. " (vague 1)

"Pas que je n'aime pas l'emploi que j’ai présentement, mais c'est juste que... j’aimerais aller plus loin dans... dans ça. Ou, sìl y avait une porte au niveau des études aussi, si vraiment il y avait quelque chose qui cliquait, je serais prêt à y aller aussi. [... S'il venait à y avoir d'autres portes ou d'autres opportunités... je les prendrais. " (vague 5)

\subsection{L'éternel étudiant}

Cette figure est associée au rêve de poursuivre un mode de vie étudiant le plus longtemps possible : études longues, formation continue et grande autonomie, par exemple dans la capacité de continuer d'étudier tout en travaillant. C'est une forme spécifique de figure identitaire, figée dans le temps présent, mais qui peut déboucher sur une carrière de professeur ou de chercheur, souvent associée à l'image de quelqu'un aux tâches variées, maître des horaires et de l'organisation du travail, tout en restant rattaché à un univers studieux. Cette figure se construit sur l'idée que les études supérieures servent d'abord à développer une culture générale, sans application pratique immédiate; elle valorise avant tout la curiosité et l'acquisition constante de nouvelles connaissances.

Elle avait déjà été repérée par Dubet (1994) dans son analyse du contexte universitaire français et semble dériver de la figure classique de l'«étudiant bohème " de classe bourgeoise qui poursuit des études en attendant de reprendre l'entreprise familiale. Au Québec, comme en France, la figure d'origine semble s'être diffusée au sein des autres classes, soutenue par un discours social favorisant les études longues mais qui ne s'appuie toutefois pas pour toujours sur un destin assuré par l'origine familiale.

L'exemple de Noémie illustre cette figure pour laquelle les études ont une valeur suprême par rapport à la possibilité présente et future de travailler. À l'amorce d'études longues en vue de devenir psychologue, elle se projette déjà en doctorat sans y voir une issue clairement professionnelle. Tout au long de l'enquête, et contrairement à la très grande majorité de ses collègues (Bidart, Bourdon et Charbonneau, 2011), elle ne travaille pas pendant l'année scolaire et s'oppose à son entourage qui la presse de le faire. Elle ne parvient pas à dépasser son aversion pour un emploi sans qualification et le seul emploi étudiant qu'elle 
occupe (sauveteuse dans un camping) exigera d'elle une formation d'un an qu'elle complètera tout en étudiant la psychologie :

"Prof c'est bien, mettons t'as une partie d'enseignement, puis t'as une partie où tu es à ton bureau et tu prépares tes cours. Ça reste quand même l'école. [...] Le travail, oui, c'est l'accomplissement de soi-même. Si je savais que j'aurai tout l'argent du monde et que jaurai pas besoin de travailler plus tard, jétudierais. " (vague 3)

\section{Concomitance et changements de figures de réussite : une autre perspective sur les bifurcations}

Ces figures de réussite ne constituent pas ici une liste exhaustive des projections, mais un ensemble de modèles récurrents dans le panel des jeunes Québécois. En même temps, ces figures existent au-delà du panel dans la mesure où des conditions et des facteurs institutionnels, culturels et sociétaux, les rendent non seulement légitimes mais également envisageables et réalisables dans les parcours effectifs des jeunes.

Un examen de la distribution des figures de réussite lors de la première vague d'entretiens du panel $(n=53)$ montre qu'un peu plus de la moitié (32) des jeunes se réferent à la première figure, celle du professionnel diplômé, ce qui n'est pas étonnant compte tenu de leur recrutement à l'amorce d'études postsecondaires. La prédominance de cette figure est cohérente avec le discours ambiant sur la " société des savoirs " qui prône l'intérêt d'une scolarisation prolongée pour faciliter l'insertion. Cette observation concorde aussi avec l'importance accordée aux études par les cégépiens, observée dans l'enquête de Roy (2006) sur les valeurs des jeunes québécois. La présence des autres figures montre toutefois la multiplicité et la variété des normes sociales qui guident les choix des jeunes : onze parmi eux se réfèrent à la figure du travailleur identitaire; six à celle du consommateur hédoniste ; quatre à la figure du travailleur en sécurité d'emploi ; deux à la figure d'éternel étudiant et deux à la figure de l'entrepreneur.

Par ailleurs, les jeunes ne se situent pas nécessairement et toujours en référence à une seule figure. Sur la durée de l'enquête, on constate une concomitance de figures sur des périodes plus ou moins longues du parcours chez cinq jeunes. Dès la première vague, des concomitances, toutes différentes, sont observées chez quatre jeunes : professionnel diplômé et consommateur hédoniste (Sarah) ; professionnel diplômé et travailleur en sécurité (Laurent) ; professionnel diplômé et éternel étudiant (Noémie) ; travailleur identitaire et entrepreneur (Julia). On voit aussi s'installer une concomitance entre les figures du travailleur identitaire et d'éternel étudiant, chez Nathan, à partir de la cinquième vague. Cette concomitance exprime un ancrage double et simultané dans des figures différentes, jusqu'à la rencontre d'un contexte qui donne davantage de poids à l'une des deux figures. Ce double ancrage peut s'articuler entre des figures complémentaires, comme pour Julia, chez laquelle cohabitent les figures du travailleur identitaire et de l'entrepreneur, qui misent toutes deux 
sur un certain individualisme, soit par l'initiative et la responsabilité, soit par l'épanouissement personnel. La concomitance peut aussi se présenter entre figures en apparence contrastées, comme chez Sarah, qui se situe en référence au professionnel diplômé pour qui le diplôme est une finalité essentielle, et au consommateur hédoniste chez qui le diplôme est seulement un moyen.

Les figures de réussite apparaissent dans l'ensemble assez stables, mais on relève tout de même onze jeunes qui changent une ou deux fois de figure de réussite au cours des cinq années du suivi longitudinal, modifiant ainsi leur rapport aux finalités et priorités en matière de formation et d'emploi.

Les liens entre les parcours et les figures permettent de nuancer l'analyse des bifurcations alors que ce qui peut apparaître, à première vue, comme des renoncements, des baisses d'ambitions ou des désorientations conduisant à des réorientations parfois radicales peut sous-tendre l'interaction entre deux figures concomitantes ou une simple modification de stratégie visant à atteindre un même but, une même figure, sans rupture dans la cohérence biographique.

Deux exemples permettent d'illustrer ces inflexions autour des figures, le premier fait de changements à la fois dans les parcours effectifs et les figures et le second semé de changements effectifs d'orientation sous-tendus par une relative stabilité dans la coexistence de deux figures de réussite.

\subsection{Des bifurcations radicales qui s'appuient sur le changement de figure de réussite}

Ainsi Mathilde, lors des premières vagues - elle est née de parents ouvriers n'ayant pas fréquenté le postsecondaire - projette d'étudier jusqu'au doctorat en psychologie et rattache son insertion future au diplôme universitaire en tant que professionnelle diplômée:

"Bien, j'aimerais ça, me rendre psychologue, faire un doctorat pour pouvoir pratiquer en psychologie." (vague 1).

Le diplôme et l'expérience scolaire ont une valeur centrale, non seulement pour exercer plus tard le métier qu'elle aime, mais également en tant que symbole de réussite pour sa famille et comme condition de l'insertion :

"J'aimerais ça, dans cinq ans ou moins, avoir commencé ma Psycho, et si possible me trouver un travail qui va me permettre de peut-être travailler avec les enfants ou des choses comme ça. [...] Puis aussi avoir le papier, le diplôme, puis aller le montrer à ma famille [...] Je serais celle qui aurait fait le plus haut niveau d'études. " (vague 2).

"Plus que ça avance, plus le monde a besoin d'être instruit. Puis je crois qu'en ayant de l'expérience théorique, tu vas être plus apte à prendre des expériences pratiques [...] Je crois que plus d'études tu as, plus c'est facile d'avoir un emploi. " (vague 3) 
Un processus de réorientation, qu'elle impute à un emploi étudiant avec son beau-père dans l'immobilier et à une admission refusée en technique d'éducation spécialisée, qu'elle conçoit comme une passerelle vers l'université, l'amène toutefois à changer radicalement sa conception de la réussite pour adopter la figure de l'entrepreneure, particulièrement éloignée de sa conception initiale. Cette expérience professionnelle transforme ses modèles et éveille son esprit d'entreprise. Le diplôme, auparavant considéré comme un passage incontournable, devient alors un moyen, une ressource accessoire pour développer un emploi autonome. Elle conçoit maintenant l'expérience de travail comme plus formatrice et ouvrant davantage de potentiel pour son insertion que la scolarisation :

"Je suis allée faire mon cours d'agent immobilier pour travailler avec mon beau-père. [...] Parce que je me voyais pas aller à l'université... je voyais pas le but là. [...] Il faut que je sois trois ans agente pour aller suivre mon cours d'agent agrée qui me permettrait d'ouvrir ma propre bannière d'agents immobiliers et de faire plus de commercial. [...] Bien, c'est sûr que j'aimerais avoir une belle carrière là. " (vague 4)

Cette nouvelle représentation de l'articulation, beaucoup moins forte, entre le diplôme et l'emploi, lui permet d'explorer plusieurs voies nouvelles. Le salaire, les possibilités d'avancement et l'autonomie deviennent des critères importants dans les choix qui orienteront son parcours. Une transformation radicale de figure de réussite s'opère chez la jeune femme, dont l'initiative personnelle et la maîtrise des possibilités font partie d'une stratégie vers l'emploi autonome :

"Je vais peut-être aller faire mon bac en Comptabilité. Parce qu'en étant agent immobilier, on est porté à faire de la comptabilité, surtout si tu veux vendre du commercial. [...] Je veux faire l'un pour favoriser l'autre. Tu sais, dans le fond, je veux être comptable agréé pour pouvoir vendre de plus grands immeubles. [...]" (vague 5)

Elle devient à la fin très ambitieuse :

"Je ne veux pas être obligée de travailler pour une compagnie. Je veux travailler pour moi. [...] Comme mon beau-père dit: "Tu vas pouvoir, un jour, vendre une école." Il y en aura peut-être plus de cégeps dans une couple d'années. J'vais peut-être être là pour le vendre. " (vague 5)

Le cas de Mathilde montre comment une bifurcation radicale du parcours est soutenue par le changement des figures de réussite, à la suite d'une fermeture (l'échec d'accès en formation) et d'une ouverture (la proposition du beau-père d'un nouveau métier) des possibilités du contexte. La disponibilité de figures de réussite alternatives dans l'environnement social lui offre l'opportunité de modifier sa conception de ce qui pourrait constituer un terme légitime à son parcours. Les référents normatifs, qui peuvent être en tension au moment du choix, laissent la place à l'expérience ; celle-ci deviendra une ressource dans les prises de décisions ultérieures et ces dernières vont renforcer la nouvelle orientation. 


\subsection{Des bifurcations effectives qui s'inscrivent dans la cohérence biographique}

On observe aussi des changements plus graduels de référents normatifs où deux figures cohabitent pendant un moment avant qu'une des deux ne perde son potentiel mobilisateur, créant une bifurcation effective du parcours. L'exemple de Laurent illustre cette situation, d'abord avec concomitance des figures de professionnel diplômé et de travailleur en sécurité, suivie d'une valorisation accrue de la sécurité et d'une diminution progressive de l'importance accordée au diplôme.

Dès la première rencontre, Laurent se destine à l'enseignement de l'histoire au secondaire, une profession qu'il associe à de bonnes conditions d'emploi : "Professeur [...] Bien, je suis quelqu'un qui aime ça, travailler avec les jeunes. Puis je trouvais qu'il y avait des belles conditions aussi là, comme deux mois de vacances puis un bon salaire et le reste. " (vague 1)

Au cours des mois suivants, ses ambitions se précisent : il se voit enseigner l'histoire militaire dans l'armée. Le diplôme apparaît toujours comme une ressource essentielle, mais les conditions d'emploi prennent aussi une place importante dans ses projections :

"Moi, j’aimerais bien ça, avoir un diplôme, mais spécialisé en histoire militaire, et être capable aussi d'enseigner aux forces armées. Enseigner au sein des Forces armées canadiennes, parce que les avantages et tout ça, c'est super bon. " (vague 2)

Lorsqu'on le rencontre en vague 4 , il a interrompu ses études et il se décrit moins emballé qu'auparavant par l'enseignement, moins convaincu de l'importance du diplôme et surtout peu épanoui dans la formation en général :

"On dirait qu'un matin je me suis levé, puis je me suis dit: Bah... Je ne sais pas ce que ça me donne de continuer. " (vague 4)

Il considère désormais le travail comme plus important en attendant de se trouver une nouvelle vocation et, surtout, il accorde une importance accrue à son expérience pratique dans l'emploi pour retrouver un nouveau métier :

"En lâchant le cégep, j’ai essayé de noter... vraiment, ce que j’aimais faire dans la vie, tu sais? [...] Bon bien, par rapport à ça, quel métier soriente vers ça?" [...] Je suis un gars qui aime ça conduire. " (vague 4)

Les offres du bureau d'Emploi-Québec, qui vont lui permettre de se faire une idée des possibilités concrètes du marché du travail, et le soutien financier et affectif de son entourage vont l'aider à mener sa réorientation vers le métier de chauffeur de bus. Il est intéressant de noter que Laurent, à ce moment, voit dans cette réorientation une grande cohérence avec ce qu'il a toujours visé : assurer son parcours à plus long terme à travers l'emploi :

"Tu sais, en plus de ça, toutes les conditions sont super bonnes, là. Tu sais, le salaire, en commençant, c'est quand même intéressant. Ils donnent $17 \$$ de l'heure. Puis après deux ans et demi, c’est 23 \$, puis après un autre deux ans et demi, tu plafonnes à 36 \$. Puis, après ça, bien, 
tu as une pension au bout de 30 ans, là. Tu as un fond de pension, puis tout ça. Moi, dans 30 ans, ça me donne 54 ans. Fait que tu sais... pour prendre ma retraite, ça n'est pas bien vieux, là!» (vague 4)

La description de l'emploi s'ajuste complètement à l'un de ses deux modèles de départ, le travailleur en sécurité, qui occupe maintenant l'avant-scène de ses référents normatifs. D'un point de vue objectif, il opère une bifurcation du parcours, mais celle-ci s'inscrit largement dans la continuité du modèle de réussite sociale au fondement de ses choix. Les passerelles de ce changement sont tant individuelles, par la stabilité des critères du jeune, que sociales, par le soutien de sa famille ou les opportunités offertes par un système d'information sur l'emploi qui permet l'exploration de voies favorables à la concrétisation de ses ambitions.

Tant les changements que la concomitance des figures de réussite au sein des parcours contribuent à étayer et nuancer l'analyse des bifurcations biographiques et à approfondir la discussion sur la nature objective/subjective de la réorientation et sur la distinction entre la perspective du chercheur et celle de l'acteur de la bifurcation. On constate que les réorientations des parcours se traduisant par des bifurcations dans le choix des métiers reposent, d'une part, sur le délaissement, le remplacement ou le renforcement des modèles de réussite qui suivent des événements personnels ou le contexte et, d'autre part, que les ruptures observées dans les pratiques ne résultent pas toujours de ruptures dans les représentations des acteurs eux-mêmes.

Les figures de réussite fonctionnent ainsi comme des principes ou des " moteurs " du changement, au sens de Pérocheau (2010), c'est-à-dire qu'elles génèrent le mouvement et l'assemblage des facteurs à l'origine des bifurcations professionnelles. Les opportunités du contexte, qui s'ouvrent ou se ferment, les expériences dans un domaine d'études ou de travail, les évaluations personnelles de l'intérêt ou de la priorité de la formation ou du travail dans leur vie viennent reconfigurer le sens de la réussite et le modèle à mettre en œuvre, à travers les figures. En tant que moteurs, les figures de réussite n'expliquent pas nécessairement le sens ultime de la bifurcation, mais leur prise en compte dans l'analyse permet de rendre intelligible sa dynamique.

\section{Conclusion}

Les figures de réussite condensent en idéaux-types des configurations de normes qui touchent à plusieurs aspects de la réussite sociale : centralité du travail ; articulation travail et vie hors travail ; importance du diplôme et de la certification ; niveau ou spécialité de la formation ; forme d'articulation entre le diplôme et l'emploi ; savoirs pratiques ou théoriques ; argent, prestige, sécurité, savoir, activité. Elles regroupent et agencent, dans une mesure unique, plusieurs dimensions qui définissent un « terme perçu comme favorable " au parcours d'insertion, orientent les choix, et offrent des critères d'évaluation de la réussite de cette insertion pour les jeunes. La notion de figures de réussite comme agencement 
de normes se définissant sur plusieurs registres permet aussi d'insister sur le fait que ces normes ne constituent pas des principes d'action isolés, mais qu'elles prennent sens au sein d'un ensemble d'autres normes (Livet, 2006) associées à un contexte historique qui les rend légitimes et praticables.

La variété constatée des figures de réussite, qui montre que plusieurs normes cohabitent au sein d'un groupe donné, fait écho aux constats de Coinaud et Vivent (2010) sur les valeurs différenciées du diplôme aux yeux des élèves engagés dans une même formation, dès lors qu'ils y sont parvenus par des étapes et des choix différents. Cette variété amène aussi à résister à la tentation de mesurer la réussite des parcours à l'aune d'une seule norme, soit scolaire, soit professionnelle, alors que des normes tout aussi fortes, celles touchant notamment à la parentalité (Poelmans, 2012), à la famille ou aux amis (Royer, Pronovost et Charbonneau, 2004) peuvent aussi y jouer un grand rôle.

Le "pluralisme normatif» constaté par Bernheim (2011) se révèle à l'aune des figures de réussite dans la construction des parcours observés ici. En contrepartie, le constat que les variations ne se multiplient pas à l'infini et l'observation de certaines récurrences ouvrent la voie à des possibilités d'explication sociologique des parcours, que ce soit du côté de la montée en généralisation, par leur association avec des catégories sociales et des particularités sociétales (Fernando et Cohen, 2014 ; Lancaster et Drasgow, 1994), ou d'un point de vue plus micro dans la mise au jour des éléments du contexte ayant concouru à leur légitimité et leur désirabilité.

Ainsi, l'inscription de cette analyse des figures de réussite dans une comparaison internationale, comme celle à laquelle nous avons participé dans le cadre du projet Bipaje, peut ouvrir sur un dépassement de la dichotomie à laquelle est souvent condamnée la sociologie de la norme, selon Demeulenaere (2003) : se limiter à un registre essentiellement descriptif pour assurer une certaine neutralité scientifique, ou se borner à des explications strictement locales et contextuelles. Cette ouverture permet ainsi de déboucher sur des registres explicatifs qui relient les caractéristiques structurelles et culturelles des contextes sociétaux et les éléments constitutifs des cibles désirables de l'insertion sociale; il s'agit ainsi de mieux comprendre comment se construisent les parcours des jeunes et leurs bifurcations.

L'analyse a aussi révélé que, malgré leur relative stabilité, les figures de réussite sont susceptibles de coexister et de changer au sein d'un même parcours, à différents moments du cheminement. Ces changements ne sont pas fortuits, même s'ils peuvent parfois être imprévus et relativement irréversibles pour le jeune. Des facteurs des différentes échelles (Grossetti, 2004) (individuelle, institutionnelle, macroéconomique, etc.) contribuent à leur évolution, se combinant pour offrir aux jeunes une gamme de représentations de réussite sociale plus ou moins adaptée à leur situation et au point où ils en sont dans leur parcours.

Les normes offrent une dimension évaluative des positions sociales et de leurs attributs, tout en comportant une dimension prescriptive ; dès lors, leur transgression est générale- 
ment conçue comme ouvrant la voie à diverses sanctions (Demeulenaere, 2003), comme le déni de reconnaissance (Honneth, 2002) ou l'exclusion. Ces opérations sont effectivement en jeu lors des choix effectués par les jeunes au cours de leur insertion ; cependant, les changements observés de figures de réussite viennent nuancer l'affirmation selon laquelle "la transgression d'une norme soulève l'inquiétude du retour à un monde fondamentalement imprévisible " (Prairat, 2012, p. 40). Il semble plutôt que la transgression des normes se pose bien plutôt en amorce de l'inscription dans un autre système de normes que comme prélude à une totale anomie. La légitimité et l'effectivité des normes se construisent ainsi pour un contexte donné et se redéfinissent au cours de l'action et de l'interaction (Munck, 1997). Cette vision contextuelle et dynamique, voire procédurale, de la norme appelle à une observation située et diachronique des parcours dans leur lien avec le social, comme celle que nous avons conduite dans notre domaine d'étude.

L'analyse longitudinale prend donc ici toute sa pertinence, car c'est à travers l'observation des temporalités relativement longues du parcours qu'il est possible de suivre ces évolutions et changements. Les données longitudinales permettent d'observer l'action des figures de réussite sur les parcours effectifs et les changements de référents normatifs. La combinaison du suivi des représentations et des pratiques effectives dans les parcours - avec ou sans changement de figure - met ainsi en évidence la non-correspondance mécanique entre normes de réussite et parcours de réussite.

Ce qui peut être jugé, de "l'extérieur ", au mieux comme une bifurcation professionnelle et au pire comme un échec scolaire ou une révision à la baisse des ambitions professionnelles, n'est pas nécessairement évalué comme tel par le jeune adulte à l'aune des autres normes sociales disponibles pour la construction du parcours. Par leur lien aux représentations des acteurs, les figures de réussite contribuent à nuancer une évaluation trop rapide, par le chercheur, de l'existence d'une bifurcation et de sa radicalité au sein des parcours effectifs. Comme nous l'avons observé, si des changements de métier sont balisés par un changement de figure de réussite, d'autres changements effectifs du parcours se produisent sans qu'il y ait changement de modèle pour le jeune.

Enfin, sur le plan conceptuel, les figures de réussite constituent le pendant des régimes d'action publique 5 (Verdier, 2010) en matière d'orientation scolaire et professionnelle. Mais alors que les régimes d'action analysent l'hétérogénéité des systèmes d'orientation, les figures permettent de mettre en lumière la diversité et les dynamiques des conceptions individuelles au regard de la réussite sociale et professionnelle. Ce croisement des points de vue et la dimension procédurale des normes sociales appellent donc à la prudence dans l'entreprise d'élaboration de quelques mesures idéelles et abstraites

5. Le concept de régimes d'action publique réfère à une construction idéal-typique des conceptions différenciées de l'action publique légitime dans un domaine particulier et aux conventions qui viennent les opérationnaliser. 
de la réussite ne tenant pas compte de la perspective des acteurs, surtout si ces mesures doivent éclairer les décisions en matière de politiques publiques. Les dispositifs facilitant la conciliation études-travail-famille pour favoriser l'obtention du diplôme prendront sens pour certains, alors que d'autres seront mieux servis par des politiques de sécurisation des parcours et des transitions, et que d'autres encore seront plus réceptifs aux incitations à l'entreprenariat et au travail autonome.

\section{Bibliographie}

Beck U. (2001), La société du risque : sur la voie d'une autre modernité, Paris, Aubier.

Bélanger P.W. (1986), "La réponse du Québec aux problèmes d'équité et d'excellence dans l'enseignement postsecondaire ", Recherches sociographiques, 27(3), pp. 65-384.

Bernheim E. (2011), «Le pluralisme normatif : un nouveau paradigme pour appréhender les mutations sociales et juridiques? ", Revue interdisciplinaire d'études juridiques Vol. 67, (2), pp. 1-41.

Bessin M., Bidart C. et Grossetti M. (2010), Bifurcations : les sciences sociales face aux ruptures et à l'événement, Paris, La Découverte.

Bidart C., Bourdon S. et Charbonneau J. (2011), " Le rapport au travail de jeunes au Québec et en France : mise en perspective longitudinale ", in Degenne A., Mary C., Moulin et Grelet Y. (dir.), Les catégories sociales et leurs frontières, Québec, Presses de l'université Laval, pp. 85-110.

Cavalli S. (2007), "Modèle de parcours de vie et individualisation ", Gérontologie et société, vol. 4, (123), pp. 55-69.

Coinaud C. et Vivent C. (2010), "Les orientations scolaires, entre tâtonnement et réappropriation ", Formation Emploi, 109, pp. 71-84.

Demeulenaere P. (2003), Les normes sociales : entre accords et désaccords, Paris, Presses universitaires de France.

Dubet F. (1994), «Dimensions et figures de l'expérience étudiante dans l'université de masse ", Revue française de sociologie, 35(4), pp. 511-532.

Fernando W.D.A. et Cohen L. (2014), "Respectable Femininity and Career Agency: Exploring Paradoxical Imperatives", Gender, Work and Organization, Vol. 21, (2), pp. 149-164.

Grossetti M. (2004), Sociologie de l'imprévisible : dynamique de l'activité et des formes sociales, Paris, Presses universitaires de France.

Honneth A. (2002), La lutte pour la reconnaissance, Paris, Les Éditions du Cerf. 
Lancaster A. et Drasgow F. (1994), "Choosing a Norm Group for Counseling: Legislation, Regulation, and Nontraditional Careers", Psychological Assessment, Vol. 6(4), pp. 313-320.

Livet P. (2006), Les normes, Paris, Armand Colin.

Longo M. E., Bourdon S., Charbonneau J., Kornig C. et Mora V. (2013), « Normes sociales et imprévisibilités biographiques : une comparaison France, Québec et Argentine ", Agora débats/ jeunesses, (65), pp. 93-108.

Munck J. de (1997), " Normes et Procédures : les coordonnées d'un débat », in Munck J. de, Verhoeven M., Les mutations du rapport à la norme : un changement dans la modernité ?, Bruxelles, De Boeck Université.

Pérocheau G. (2010), «Les moteurs, principes génératifs du mouvement dans les processus ", in Mendez A., Processus : concepts et méthode pour l'analyse temporelle en sciences sociales, Louvain-la-Neuve, Academia-Bruylant, pp. 123-139.

Poelmans S. (2012), "The "Triple-N” Model: Changing Normative Beliefs about Parenting and Career Success", Journal of Social Issues, Vol. 68 (4), pp. 838-847.

Prairat E. (2012), "Considérations sur l'idée de norme », Les Sciences de l'éducation - Pour l'Ėre nouvelle, vol. 45 (1), pp. 33-50.

Roy J. (2006), "Les valeurs des cégépiens et la réussite scolaire : portrait des valeurs et repères pour l'intervention ", Service social, vol. 52 (1), pp. 31-46.

Royer C., Pronovost G. et Charbonneau S. (2004), «Valeurs sociales fondamentales des jeunes québécoises et québécois : ce qui compte pour eux ", in Pronovost G. et Royer C. (Dir.)., Les valeurs des jeunes, Presses de l'université du Québec, pp. 50-68.

Sen A. (2000), Repenser l'égalité (Trad. par P. Chemla), Paris, Seuil (1 $1^{\text {re }}$ éd Inequality Reexamined).

Verdier É. (2010), «L'orientation scolaire et professionnelle : entre assignation et idées floues, l'anarchie organisée ", Formation Emploi, n 109, pp. 113-126. 


\section{L'ORIENTATION SCOLAIRE ET PROFESSIONNELLE}

Revue de l'Institut national d'étude du travail et d'orientation professionnelle

Numéro 45/2 (juin 2016)

Numéro thématique - Anne-Marie Costalat-Founeau (Coord.)

Identité sociale et dynamique du projet

\section{Anne-Marie Costalat-Founeau \& Even Loarer \\ Introduction}

\section{Isabelle Faurie \& Anne-Marie Costalat-Founeau}

Sentiments d'efficacité personnelle et dynamique capacitaire dans les choix d'orientation atypique

\section{Philippe Thorel, Anne-Marie Vonthron, Émilie Vayre}

Dynamique identitaire, tonalité affective et sentiment d'efficacité au retour d'expatriation professionnelle : le cas de cadres réintégrant leur organisation d'origine

Imane Zineb Lahrizi, Jonas Masdonati, Isabelle Skakni \& Geneviève Fournier Entre attentes et réalité : la construction de l'identité professionnelle des diplômée-s de la formation professionnelle et technique

\section{Joëlle Mezza, Dominique Lhuilier \& Even Loarer}

Réorganisation du projet professionnel et remaniement identitaire : les effets capacitaires de l'activité des personnes atteintes de maladies chroniques

\section{Ghislain Mary \& Anne-Marie Costalat-Founeau}

Exploration de la dynamique identitaire en situation d'accompagnement au projet 